Article

\title{
Transmission of SARS-CoV-2 in Northern Ghana; Insights from Whole Genome Sequencing
}

\author{
Augustina Angelina Sylverken 1,2,*, Philip El-Duah 1,3, Michael Owusu 1,4, Julia Schneider ${ }^{3}$, \\ Richmond Yeboah 1, Nana Kwame Ayisi-Boateng 5, Richmond Gorman 1, Eric Adu 1, Alexander \\ Kwarteng 1,6, Michael Frimpong 1,7, Sherihane Aryeetey 1, Jesse Addo Asamoah 1, Yaw Ampem \\ Amoako 5, John Humphrey Amuasi 1,8, Jörn Beheim-Schwarzbach 3, Ellis Owusu-Dabo ${ }^{8}$, Yaw \\ Adu-Sarkodie ${ }^{9}$, Kwasi Obiri-Danso ${ }^{2}$, Victor Max Corman ${ }^{3}$, Christian Drosten ${ }^{3}$ and Richard \\ Phillips 1,5
}

1 Kumasi Centre for Collaborative Research in Tropical Medicine, PMB, UPO, Kumasi 00233, Ghana; philip.el-duah@charite.de (P.E.-D.); michaelowusu80@gmail.com (M.O.); yeboahrichmond82@yahoo.com (R.Y.); richgormann3@gmail.com (R.G.); e.edu@kccr.de (E.A.); akwarteng@knust.edu.gh (A.K.); frimpong@kccr.de (M.F.); asherihanne@gmail.com (S.A.); jesseasamoah1@gmail.com (J.A.A.); amuasi@kccr.de (J.H.A.); phillips@kccr.de (R.P.)

2 Department of Theoretical and Applied Biology, Kwame Nkrumah University of Science and Technology, Kumasi, Ghana; obirid@yahoo.com

3 Institute of Virology, Charite, Universitätsmedizin Berlin, Germany; julia.schneider@charite.de (J.S.); joern.beheim-schwarzbach@charite.de (J.B.-S.); victor.corman@charite.de (V.M.C.); christian.drosten@charite.de (C.D.)

4 Department of Medical Diagnostics, Kwame Nkrumah University of Science and Technology, Kumasi, Ghana

5 Department of Medicine, Kwame Nkrumah University of Science and Technology, Kumasi, Ghana; ayisi31@gmail.com (N.K.A.-B.); yamoako2002@gmail.com (Y.A.A.)

6 Department of Biochemistry and Biotechnology, Kwame Nkrumah University of Science and Technology, Kumasi, Ghana

7 Department of Molecular Medicine, Kwame Nkrumah University of Science and Technology, Kumasi, Ghana

8 Department of Global and International Health, Kwame Nkrumah University of Science and Technology, Kumasi, Ghana; owusudabo@yahoo.com

9 Department of Clinical Microbiology, Kwame Nkrumah University of Science and Technology, Kumasi, Ghana; yasax@hotmail.co.uk

* Correspondence: asylverken@knust.edu.gh; +233 244214625

Received: date; Accepted: date; Published: date

\begin{abstract}
In regions lacking genomic data, analysis of sequences from the early stages of an outbreak can provide important insights into the diversity of pathogens present. Following the detection of the first imported case of COVID-19 in the Northern sector of Ghana on 13 $3^{\text {th }}$ March 2020, we have now molecularly characterized and phylogenetically analysed sequences including three (3) complete genomes of the severe acute respiratory syndrome coronavirus 2 (SARS-CoV-2) isolated from nine (9) patients observed in Ghana. Eight (8) of these patients reported with a recent history of foreign travel and one (1) with no history of foreign travel. We performed high throughput sequencing for 9 samples following the determination of high concentration of viral RNA. In addition, we estimated the potential impact that long distance transportation of samples to testing centres may have on sequencing outcomes. Here, two samples that were closest in terms of viral RNA concentration but transported from sites which are over $400 \mathrm{~km}$ apart were assessed. All sequences were compared to previous sequences from Ghana and representative sequences from regions where our patients had previously travelled. Complete genomes were obtained for three (3) sequences and with another near complete genome with a coverage of $95.6 \%$. Sequences with coverage in excess of $80 \%$ were found to belong to three lineages namely A, B.1 and B.2. Our
\end{abstract}


sequences clustered in two different clades with the majority falling within a clade composed of sequences from sub-Saharan Africa. Less RNA fragmentation was seen in sample KATH23 which was collected $9 \mathrm{~km}$ compared with sample TTH6 which was collected and transported over a distance of $400 \mathrm{~km}$ to the testing site. The clustering of several sequences from sub-Saharan Africa suggests regional circulation of the viruses in the subregion. Importantly, there may be the need to decentralize testing sites and build more capacity across Africa to boost the sequencing output of the subregion.

Keywords: Ghana; SARS-CoV-2; transmission; Phylogenetics

\section{Introduction}

Coronaviruses (CoVs) are minute in size $(65-125 \mathrm{~nm}$ in diameter) and contain a single-stranded RNA with size ranging from 26 to $32 \mathrm{kbs}$ in length. Subgroups of coronaviruses family are alpha $(\alpha)$, beta $(\beta)$, gamma $(\gamma)$ and delta $(\delta)$. Until 2002, when the world witnessed a severe acute respiratory syndrome (SARS) outbreak caused by SARS-CoV in Guangdong, China, coronaviruses with the exception of 229E and OC43 were thought to infect only animals [1]. Only a decade later, another pathogenic coronavirus, known as Middle East respiratory syndrome coronavirus (MERS-CoV) caused an endemic outbreak in Middle Eastern countries [2].

In December 2019, the World Health Organization (WHO) was informed about a cluster of patients who presented with pneumonia of unknown aetiology in the city of Wuhan (Hubei province) in China [3]. Shortly afterwards, a new type of coronavirus, now termed Severe Acute Respiratory Syndrome-Coronavirus-2 (SARS-CoV-2), was isolated and identified by Scientists from China [4]. Sequencing results revealed that it belongs to the $\beta$-coronavirus genus of the Coronaviridae family, and has $96 \%$ genomic identity with a previously detected SARS-like bat coronavirus [5], [6]. The genetic sequence of this new virus was shared with the international community on $10^{\text {th }}$ January 2020 [7], [8]. The infection caused by this virus has spread to all the WHO regions and has contributed adversely to an enormous global impact. Since then, it became a public health emergency of international concern and on March 11, 2020, the World Health Organization (WHO) declared it a global pandemic [9]. This action by WHO prompted an increased and sustained international action and response. As of $27^{\text {th }}$ August, 2020 over 24 million cases were confirmed with more than 830,000 deaths globally (https://www.worldometers.info/coronavirus/).

The first confirmed case of COVID-19 in Africa was reported from Egypt on $14^{\text {th }}$ February 2020 [10] and in sub-Saharan Africa from Nigeria on $28^{\text {th }}$ February 2020 [11]. Ghana recorded its first confirmed case of COVID-19 on $12^{\text {th }}$ March 2020 [12]. Following this, all suspected cases of COVID19 were confirmed by reverse transcription-polymerase chain reaction (RT-PCR) as recommended by the WHO [13]. While RT-PCR still remains the gold standard, issues relating to transportation or shipping of samples from primary healthcare facilities to centralised testing laboratories, laboratory infrastructure, human resources, supply chain management and stockpile of laboratory consumables/reagents have remained key challenges [14]-[16]. Beyond using RT-PCR to establish the presence of the virus, one big shortfall in several African countries is the inability to sequence the virus and to understand the biology of the virus. This may partly underline deficits in understanding the distribution of the various SARS-CoV-2 strains circulating on the African continent as well as the lack of knowledge in its transmission dynamics [17].

The paucity of data is evidenced by the measure and proportion of sequences and genome data deposited on the Global Initiative on Sharing Avian Flu Data (GISAID) repository originating from Africa institutions (https://www.gisaid.org/). This means that Africa needs to up its game and build more capacity with respect to genome sequencing. Such a measure has a lot of advantages. This will help us understand the distribution of the various strains circulating on the continent and an understanding of its transmission dynamics. This could eventually assist in the development of vaccine against COVID-19. Chances are that the African sequences maybe relatively widely 
distributed within the global sequences [18]. In an effort to contribute to this initiative and following the detection of the first imported case in the Northern sector of Ghana on $13^{\text {th }}$ March 2020, we have now molecularly characterized and phylogenetically analyzed sequences including three (3) complete genomes of SARS-CoV-2 isolated from nine (9) out of the first 200 patients observed in Ghana. Eight (8) of these patients reported with a recent history of foreign travel and one without such history.

\section{Materials and Methods}

\section{Sample collection and transportation}

Between $29^{\text {th }}$ February and $28^{\text {th }}$ March 2020, samples were obtained from patients with suspected COVID-19 from the Tamale Teaching Hospital (TTH) in the Northern Region, Komfo Anokye Teaching Hospital (KATH) and Kumasi South Hospital (KSH) in the Ashanti Region of Ghana (Figure 1). Recruitment of cases at these facilities was done according to Ghana National Surveillance Strategy protocol [19]. Suspected COVID-19 cases were defined as individuals presenting with fever $\left(>38^{\circ} \mathrm{C}\right)$, or a history of fever and symptoms of respiratory tract illness such as cough, shortness of breath or individuals in close contact with a person who is under investigation or confirmed for COVID-19.

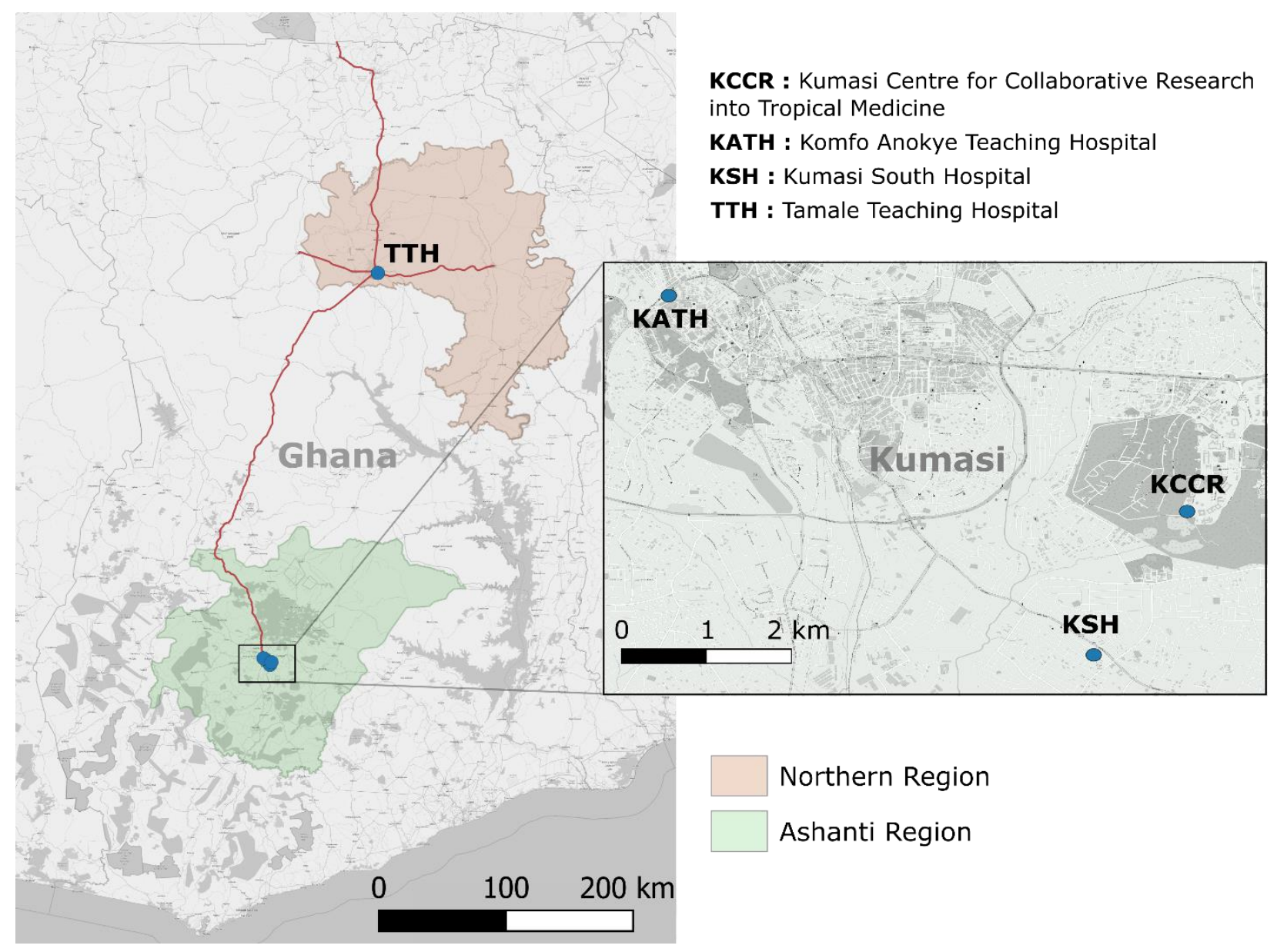

Figure 1. Map of Ghana showing sample collection sites: Map was generated using Quantum GIS version 3.6.2 and data freely available from www.openstreetmaps.org. Samples were collected from the Northern region (orange) and the Ashanti region (green) and tested at the KCCR also in Kumasi in the Ashanti region of Ghana. 
Nasopharyngeal and or oropharyngeal swabs were obtained with flocked swabs (Copan Group, Brescia, Italy) and kept in 500ml RNAlater (Qiagen, Hilden, Germany) in 1.5ml tubes (Eppendorf, Regensburg, Germany) and transported immediately for confirmation at the Kumasi Centre for Collaborative Research in Tropical Medicine (KCCR), Kumasi in the Ashanti Region of Ghana.

The KCCR is one of the two main research laboratories designated in Ghana for COVID-19 testing. This has been because of its longstanding experience and the expertise of its scientists in studies related to Coronaviruses (http://kccr.org/). The Centre is currently Ghana's second largest testing site which serves the northern sector of the country. During the early phase of the pandemic, the Centre received samples from 12 out of 16 regions in Ghana and tested approximately 1,200 samples on a daily basis.

\section{Viral RNA extraction and PCR detection}

Both nasopharyngeal and or oropharyngeal swabs from each patient were extracted together as a single sample using the Qiagen Viral RNA mini kit spin protocol (Qiagen, Hilden, Germany) according to manufacturer's instructions. Samples were eluted in a $100 \mu \mathrm{l}$ volume and detection of SARS-CoV-2 viral RNA performed using the RealStar® SARS-CoV-2 RT-PCR Kit (Altona, Germany) according to manufacturer's instructions. Sample quantification was done using an externally generated standard curve based on serially diluted, SARS-CoV-2 in vitro transcripts. All samples with cycling threshold $(\mathrm{Ct})$ of 40 and above were considered as negative. All amplification runs were validated by the inclusion of positive and negative controls.

\section{Whole genome sequencing}

High throughput sequencing for samples with sufficiently high RNA concentrations as determined by quantitative real-time PCR were sequenced using the Illumina NextSeq platform (Illumina, San Diego, California, U.S.) and the KAPA RNA Hyper Prep kit (Roche Molecular Diagnostics, Basel, Switzerland) according to manufacturer's instructions. In order to estimate the potential impact that long distance transportation of samples to testing centers may have on sequencing outcomes, two samples that were closest in terms of viral RNA concentration were assessed on the Agilent 4200 TapeStation system (Agilent Technologies, CA, USA). These samples comprised one from TTH in the North which is approximately 400 kilometers to the testing site. The other sample was from KATH in Kumasi (same city as the testing; approximately $9 \mathrm{~km}$ to the testing site).

\section{Phylogenetic analysis}

Sequences in this study with the exception of three which had less than $80 \%$ of their genomes available were compared to previous sequences from Ghana and representative sequences from regions where patients had previously travelled. These included sequences from the United States of America (USA), Japan, France and Guinea and available sequences from sub-Saharan Africa. All sequences from the region of interest as of April 2020 were obtained from GISAID (https://www.gisaid.org/) and duplicates removed. The non-redundant sequences were then clustered at a minimum threshold of $99.9 \%$ using CD-HIT (http://weizhongli-lab.org/cd-hit/) and representative sequences from each cluster selected. Multiple sequence alignments were done using the MAFFT plugin in Geneious prime (http://www.geneious.com). Phylogenetic analysis was done by Bayesian inference using the MrBayes [20] plugin in Geneious prime with a chain length of 1.1 million and a subsampling frequency of 200. A general time reversible substitution model with a gamma distribution and proportion of invariable sites $(G T R+G+I)$ was used for the analysis. All sequences with genome coverage greater than $80 \%$ were run through the Phylogenetic Assignment of Named Global Outbreak Lineages (PANGOLIN) online resource (https://pangolin.cog-uk.io/) for lineage assignment. 
Ethical approval for this work was sought from the Committee on Human Research Publications and Ethics of the School of Medicine and Dentistry at the Kwame Nkrumah University of Science and Technology (CHPRE/AP/462/19) and the Institutional Review Board of the Ghana Health Service (GHS-ERC087/03/20).

\section{Results}

\section{Sample description and comparison}

A total of nine (9) samples obtained from 9 patients that tested positive for SARS-CoV-2 were assessed. Six (6) of the samples were from the Northern region while three (3) were from the Ashanti Region of Ghana. Patients were either asymptomatic (six) or symptomatic (three) presenting with cough, headache, general weakness, sore throat, shortness of breath and diarrhea. With the exception of one, all other patients had a history of travel to either the USA, Japan, France or Guinea (Table 1).

Despite a difference of approximately only 576 copies/ $\mu$ L between Samples KATH23 and TTH6 (Table 1), TapeStation analysis of the two samples showed less RNA fragmentation in KATH23 (Supplementary Figures S1 and S2) which yielded a subsequent complete genome on first try compared to TTH6 which yielded a sequence with approximately $82.8 \%$ genome coverage going up to $95.6 \%$ with resequencing efforts. 
1 of 11

Table 1. Description of SARS-CoV-2 positive samples analyzed in this study.

\begin{tabular}{|c|c|c|c|c|c|c|}
\hline Sample ID & $\begin{array}{c}\text { Sampling } \\
\text { site }\end{array}$ & Region & Gender & Travel history & Symptoms at presentation & $\begin{array}{c}\text { Viral RNA } \\
\text { concentration } \\
(\text { Copies } / \mu \mathrm{L})\end{array}$ \\
\hline SARS-CoV-2/Tamale/TTH7/2020 & TTH & Northern & $\mathrm{F}$ & Guinea & Asymptomatic & $1.90 \times 10^{2}$ \\
\hline SARS-CoV-2/Kumasi/KSH54/2020 & $\mathrm{KSH}$ & Ashanti & $\mathrm{F}$ & USA & $\begin{array}{l}\text { General weakness, sore throat, } \\
\text { shortness of breath, diarrhea }\end{array}$ & $7.34 \times 10^{-1}$ \\
\hline SARS-CoV-2/Tamale/TTH9/2020 & TTH & Northern & $\mathrm{F}$ & Guinea & Asymptomatic & $5.25 \times 10^{0}$ \\
\hline SARS-CoV-2/Tamale/TTH10/2020 & TTH & Northern & $\mathrm{F}$ & Guinea & Asymptomatic & $6.59 \times 10^{5}$ \\
\hline SARS-CoV-2/Kumasi/KSH61/2020 & $\mathrm{KSH}$ & Ashanti & $\mathrm{M}$ & Japan, France & Cough and headache & $2.91 \times 10^{1}$ \\
\hline SARS-CoV-2/Tamale/TTH6/2020 & TTH & Northern & $\mathrm{F}$ & Guinea & Asymptomatic & $6.24 \times 10^{2}$ \\
\hline SARS-CoV-2/Tamale/TTH11/2020 & TTH & Northern & $\mathrm{F}$ & Guinea & Asymptomatic & $1.38 \times 10^{2}$ \\
\hline SARS-CoV-2/Tamale/TTH8/2020 & TTH & Northern & $\mathrm{F}$ & Guinea & Asymptomatic & $6.16 \times 10^{-1}$ \\
\hline $\begin{array}{c}\text { SARS-CoV- } \\
\text { 2/Kumasi/KATH23/2020 }\end{array}$ & KATH & Ashanti & M & No travel history & Shortness of breath & $1.20 \times 10^{3}$ \\
\hline
\end{tabular}

TTH: Tamale Teaching Hospital; KSH: Kumasi South Hospital; KATH: Komfo Anokye Teaching Hospital 
Sequence description and phylogenetic analysis

The percentage coverage of available genomes in this study ranged from as low as $23.2 \%$ to complete coverage. Complete genomes were obtained for three (3) sequences and with another near complete genome with a coverage of $95.6 \%$. Sequences with coverage in excess of $80 \%$ were found to belong to three lineages namely A, B.1 and B.2. The least number of nucleotide substitutions by comparison to the original SARS-CoV-2 genome originating in China (NC_045512) was the one with no known travel history (KATH23). The one with the most nucleotide substitutions was the one known to have travelled through at least two locations in Asia and Europe before arriving in Ghana (KSH61). The D614G amino acid substitution in the spike protein (A23403G) purported to enhance viral infectivity [21] was also found in three (3) sequences from this study (Table 2). Our sequences clustered in two different clades with the majority falling within a clade composed solely of sequences from sub-Saharan Africa (Figure 2). All sequences with greater than $50 \%$ coverage were submitted to the SARS-CoV-2 repository on GISAID platform and assigned accession numbers EPI_ISL_515181-515184 and EPI_ISL_515247-515249.

Table 2. Phylogenetic lineage description and nucleotide substitutions of available genomes in comparison to an early genome from China.

\begin{tabular}{|c|c|c|c|}
\hline Sample ID & $\begin{array}{l}\text { Percentage } \\
\text { genome } \\
\text { coverage }\end{array}$ & $\begin{array}{c}\text { Nucleotide } \\
\text { variations }\end{array}$ & $\begin{array}{c}\text { Pangolin lineage } \\
\text { (Probability) }\end{array}$ \\
\hline SARS-CoV-2/Ghana/Tamale_TTH7/2020 & 84.7 & $\begin{array}{l}\text { A14253G } \\
\text { T22714C } \\
\text { G25606T } \\
\text { T28144C }\end{array}$ & $\mathrm{A}(0.55)$ \\
\hline SARS-CoV-2/Kumasi/KSH54/2020 & 23.2 & $\begin{array}{c}\text { A1749T } \\
\text { A23403G } \\
\text { G25606T } \\
\text { T28144C }\end{array}$ & - \\
\hline SARS-CoV-2/Tamale/TTH9/2020 & 48.3 & $\begin{array}{c}\text { T380C } \\
\text { C14408T } \\
\text { G25606T } \\
\text { T28144C }\end{array}$ & - \\
\hline SARS-CoV-2/Tamale/TTH10/2020 & Complete & $\begin{array}{l}\text { G25606T } \\
\mathrm{T} 28144 \mathrm{C}\end{array}$ & A $(0.99)$ \\
\hline SARS-CoV-2/Kumasi/KSH61/2020 & 82.7 & $\begin{array}{l}\text { A12121T } \\
\text { C14408T } \\
\text { G16027T } \\
\text { T19920G } \\
\text { T22385C } \\
\text { A23403G } \\
\text { G25563T } \\
\text { G25606T } \\
\text { T28564C }\end{array}$ & B.1 (1) \\
\hline SARS-CoV-2/Tamale/TTH6/2020 & 95.6 & $\begin{array}{l}\text { G25606T } \\
\mathrm{T} 28144 \mathrm{C}\end{array}$ & B. $2(0.98)$ \\
\hline SARS-CoV-2/Tamale/TTH11/2020 & Complete & $\begin{array}{l}\text { G25606T } \\
\text { T28144C }\end{array}$ & $\mathrm{A}(0.99)$ \\
\hline SARS-CoV-2/Tamale/TTH8/2020 & 60.7 & $\begin{array}{l}\text { C14408T } \\
\text { A23403G }\end{array}$ & - \\
\hline
\end{tabular}




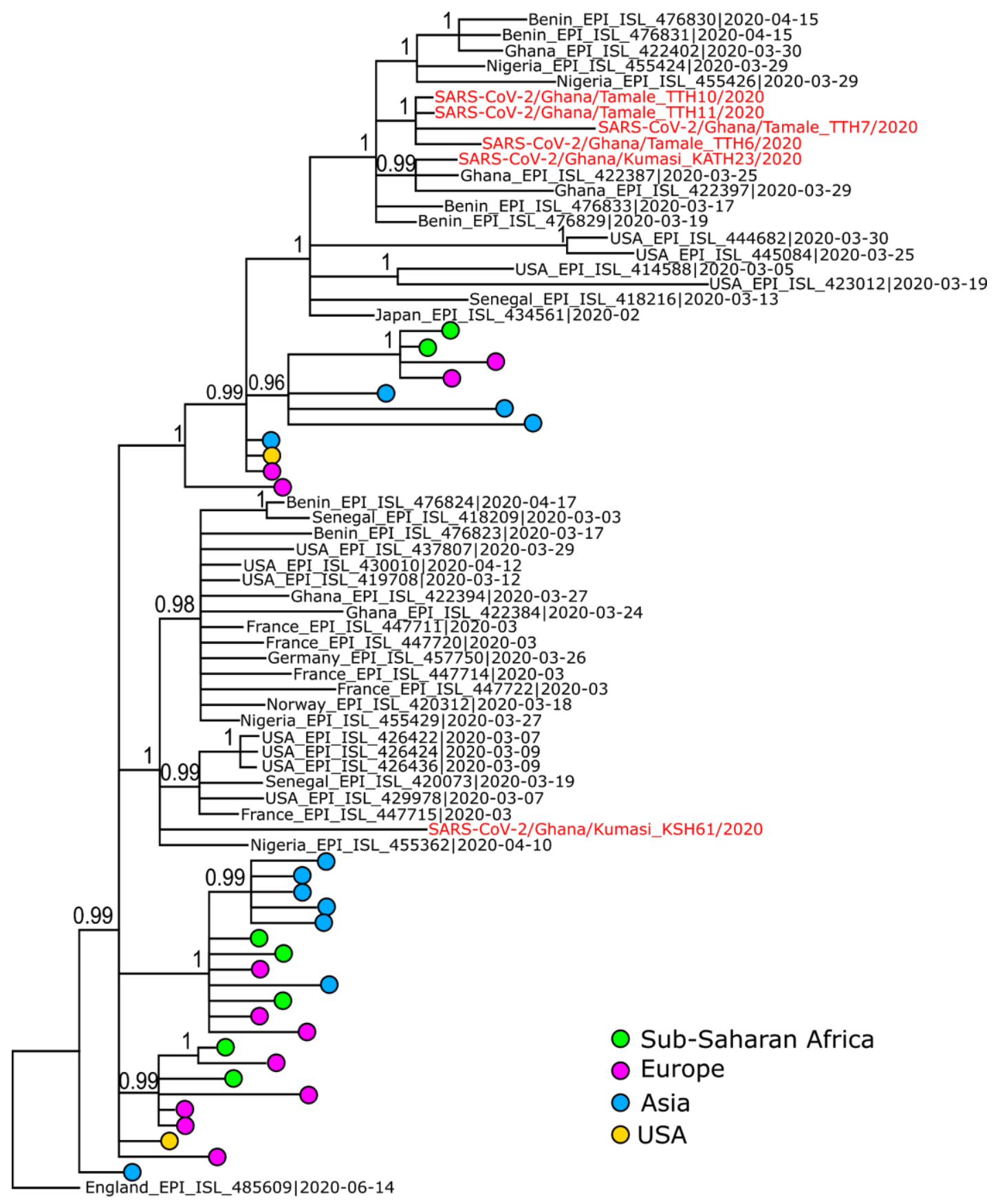

\section{$1.0 \mathrm{E}-4$}

Figure 2. Phylogenetic comparison of SARS-CoV-2 genomes obtained in the study.

Phylogenetic analysis was performed on 76 representative genomes by Bayesian inference using the GTR+G+I substitution model. Sequences in the tree are designated by location, GISAID accession numbers and date of collection. Sequences from this study are highlighted in red with isolate-specific names. The tree was rooted with randomly selected sequences from England collected in June 2020.

\section{Discussion}

Despite the impressive drive from different regions of the globe including Africa to sequence SARS-CoV-2 genomes for studying the epidemiology of the virus, Africa still lags behind in terms of 
quota of genome output as seen by a search through the SARS-CoV-2 repository on GISAID as of August 2020. Apart from lack of availability of sequencing technology and expertise which could account for this shortfall [22], contributory factors may be degradation of genetic material during transportation to available diagnostic centres due to a mostly centralized testing system prevalent on the African continent [23]. Our study shows that although two samples had similar viral loads, the samples obtained from Kumasi (approximately $9 \mathrm{~km}$ from the testing laboratory) showed less fragmentation than the one from Tamale in the northern part of Ghana and had to be transported over a long distance of approximately $400 \mathrm{~km}$ ) across the country before being processed. This highlights the importance of correct sample storage and transport conditions. Although some level of viral RNA fragmentation can be tolerated when diagnostic real-time PCR is used due to short amplicon sizes, the same cannot be said for downstream whole genome sequencing [24]. Therefore, decentralization of diagnostic centres and proper sample storage are important to boost the sequencing output of the subregion. In addition, as the world races towards development of a vaccine, the importance of sequence data from the African region cannot be overemphasized. Capacity building in Africa for this purpose is paramount.

The assigned pangolin lineages are reflective of the sampling times of the sequences as seen with the early circulating A, B.1 and B.2 lineages from January to May 2020 [25]. This supports the reported epidemiological links in terms of travel which was associated with early introduction of the virus into Ghana mainly from Asia and Europe. The sequence from the individual with no known travel history had the least number of nucleotide substitutions compared to the original sequence from China. This may hint at earlier introductions and possible community spread than previously believed.

Sequences from sub-Saharan Africa including previous ones from Ghana were observed clustering in different clades across the topology of the tree. However the subset of sequences clustering together only from sub-Saharan Africa may point to the circulation of viruses within the region due to movement through porous land borders [26], [27]. A lot of emphasis is placed on introductions from other regions like Europe and Asia through air travel but introductions from within the subregion also appears to play a major role in virus circulation through movement of people. The number of sequences included in the analysis were however limited and interpretation of this outcome should therefore be done with caution.

\section{Conclusions}

Analysis of sequences from the early stages of the outbreak can provide important insights into viral diversity present in regions lacking genomic data. There is the need for adequate sample storage and transportation when testing facilities are far from sample collection sites. The clustering of several sequences from sub-Saharan Africa suggests regional circulation of the viruses in subregion.

Author Contributions: Conceptualization, AAS, MO and RP; methodology, PE-D, JS, JBS, VMC and CD; formal analysis, AAS, MO, PE-D, RY, NKAB, RG, EA, AK, MF, SA, JAA, YAA and JHA; X.X.; resources, CD and RP; data curation, JAA; writing - original draft preparation, AAS and PE-D.; writing - review and editing, YAA, EOD, YAS and KOD; funding acquisition, CD and RP. All authors have read and agreed to the published version of the manuscript.

Funding: This research received no external funding. However, AAS, P-ED, ROP and CD are on the PANDORAID-NET (EDCTP Reg/Grant RIA2016E-1609), funded by the European and Developing Countries Clinical Trials Partnership (EDCTP2) programme which is supported under Horizon 2020, the European Union's Framework Programme for Research and Innovation. The views and opinions of authors expressed herein do not necessarily state or reflect those of EDCTP.

Acknowledgments: We thank the hospitals for allowing this study to be undertaken in their various facilities. We also appreciate the support of all healthcare workers who helped with sampling and recruitment. We appreciate the efforts of Technicians at Kumasi Centre for Collaborative Research in Tropical Medicine and the Institute of Virology, Charite, Universitätsmedizin Berlin, Germany, in supporting this work with their expertise.

Conflicts of Interest: Declare conflicts of interest or state "The authors declare no conflict of interest." 


\section{References}

[1] N. S. Zhong et al., 'Epidemiology and cause of severe acute respiratory syndrome (SARS) in Guangdong, People's Republic of China, in February, 2003', Lancet, vol. 362, no. 9393, pp. 1353-1358, 2003, doi: https://doi.org/10.1016/S0140-6736(03)14630-2.

[2] N. Wang et al., 'Structure of MERS-CoV spike receptor-binding domain complexed with human receptor DPP4', Cell Res., vol. 23, no. 8, pp. 986-993, 2013.

[3] R. Lu et al., 'Genomic characterisation and epidemiology of 2019 novel coronavirus: implications for virus origins and receptor binding', Lancet, vol. 395, no. 10224, pp. 565-574, 2020.

[4] L. Yang et al., 'Novel SARS-like Betacoronaviruses in Bats, China, 2011', Emerg Infect Dis, vol. 19, no. 6, 2013, doi: 10.3201/eid1906.121648.

[5] D. Paraskevis, E. G. Kostaki, G. Magiorkinis, G. Panayiotakopoulos, G. Sourvinos, and S. Tsiodras, 'Fullgenome evolutionary analysis of the novel corona virus (2019-nCoV) rejects the hypothesis of emergence as a result of a recent recombination event', Infect. Genet. Evol., vol. 79, p. 104212, 2020.

[6] P. Zhou et al., 'A pneumonia outbreak associated with a new coronavirus of probable bat origin. Nature. Published online February 3, 2020’. 2020.

[7] N. Zhu et al., 'A novel coronavirus from patients with pneumonia in China, 2019', N. Engl. J. Med., 2020.

[8] C. Shan et al., 'Infection with novel coronavirus (SARS-CoV-2) causes pneumonia in Rhesus macaques', Cell Res., pp. 1-8, 2020.

[9] D. Cucinotta and M. Vanelli, 'WHO declares COVID-19 a pandemic.', Acta bio-medica Atenei Parm., vol. 91, no. 1, pp. 157-160, 2020.

[10] M. Gilbert et al., 'Preparedness and vulnerability of African countries against importations of COVID19: a modelling study', Lancet, vol. 395, no. 10227, pp. 871-877, 2020.

[11] A. E. Hilda, O. E. Kolawole, A. B. Olufemi, T. Y. Senbadejo, O. M. Oyawoye, and O. J. Kola, 'Phyloevolutionary analysis of SARS-CoV-2 in Nigeria', New microbes new Infect., p. 100717, 2020.

[12] J. K. K. Asamoah, M. A. Owusu, Z. Jin, F. T. Oduro, A. Abidemi, and E. O. Gyasi, 'Global stability and cost-effectiveness analysis of COVID-19 considering the impact of the environment: using data from Ghana', Chaos, Solitons \& Fractals, p. 110103, 2020.

[13] W. H. Organization, 'Laboratory testing for coronavirus disease 2019 (COVID-19) in suspected human cases: interim guidance, 2 March 2020', World Health Organization, 2020.

[14] M. Senghore, M. K. Savi, B. Gnangnon, W. P. Hanage, and I. N. Okeke, 'Leveraging Africa's preparedness towards the next phase of the COVID-19 pandemic', Lancet Glob. Heal., 2020.

[15] F. Kobia and J. Gitaka, 'COVID-19: Are Africa's diagnostic challenges blunting response effectiveness?'. AAS Open Res., vol. 3, 2020.

[16] Y. A. Adebisi, G. I. Oke, P. S. Ademola, I. G. Chinemelum, I. O. Ogunkola, and D. E. Lucero-Prisno III, 'SARS-CoV-2 diagnostic testing in Africa: needs and challenges', Pan Afr. Med. J., vol. 35, no. Suppl 2, 2020.

[17] S. M. Garba, J. M. S. Lubuma, and B. Tsanou, 'Modeling the transmission dynamics of the COVID-19 Pandemic in South Africa', Math. Biosci., p. 108441, 2020.

[18] M. Allam, A. Ismail, Z. T. H. Khumalo, and S. Kwenda, 'Whole-Genome Sequence of the Severe Acute Respiratory Syndrome Coronavirus 2 (SARS-CoV-2)'. 
[19] 'Ghana Health Service. Ghana National Strategy Surveillance', 2020.

[20] J. P. Huelsenbeck and F. Ronquist, 'MrBayes: a Program for the Bayesian Inference of Phylogeny, v. 3.1. 2', Rochester New York, 2005.

[21] B. Korber et al., 'Tracking changes in SARS-CoV-2 Spike: evidence that D614G increases infectivity of the COVID-19 virus', Cell, 2020.

[22] M. Helmy, M. Awad, and K. A. Mosa, 'Limited resources of genome sequencing in developing countries: challenges and solutions', Appl. Transl. genomics, vol. 9, pp. 15-19, 2016.

[23] J. Nkengasong, 'Let Africa into the market for COVID-19 diagnostics', Nature, vol. 580, no. 7805, p. 565, 2020 .

[24] K. Lewandowski et al.,'The effect of nucleic acid extraction platforms and sample storage on the integrity of viral RNA for use in whole genome sequencing', J. Mol. Diagnostics, vol. 19, no. 2, pp. 303-312, 2017.

[25] A. Rambaut et al., 'A dynamic nomenclature proposal for SARS-CoV-2 to assist genomic epidemiology', bioRxiv, 2020.

[26] A. K. Sarfo and S. Karuppannan, 'Application of geospatial technologies in the covid-19 fight of Ghana', Trans. Indian Natl. Acad. Eng., pp. 1-12, 2020.

[27] R. D. Merrill et al., 'Responding to communicable diseases in internationally mobile populations at points of entry and along porous borders, Nigeria, Benin, and Togo', Emerg. Infect. Dis., vol. 23, no. Suppl 1, p. S114, 2017.

(C) 2020 by the authors. Submitted for possible open access publication under the terms and conditions of the Creative Commons Attribution (CC BY) license (http://creativecommons.org/licenses/by/4.0/). 